\title{
HYPERKÄHLER MANIFOLDS FROM THE TITS-FREUDENTHAL MAGIC SQUARE
}

\author{
ATANAS ILIEV, LAURENT MANIVEL
}

\begin{abstract}
We suggest a way to associate to each Lie algebra of type $G_{2}, D_{4}, F_{4}$, $E_{6}, E_{7}, E_{8}$ a family of polarized hyperkähler fourfolds, constructed as parametrizing certain families of cycles of hyperplane sections of certain homogeneous or quasihomogeneous varieties. These cycles are modeled on the Legendrian varieties studied by Freudenthal in his geometric approach to the celebrated Tits-Freudenthal magic square of Lie algebras.
\end{abstract}

\section{INTRODUCTION}

Hyperkähler manifolds, also called irreducible compact holomorphic symplectic varieties, are a notoriously mysterious and challenging class of varieties. A very frustrating question is to decide whether there could exist many examples besides deformations of Hilbert schemes $\mathrm{Hilb}_{n}(S)$ of points on a K3 surface $S$, and the closely related generalized Kummer varieties. Even the general deformation of $\operatorname{Hilb}_{n}(S)$ is not known. If we had a polarization and consider deformations of polarized holomorphic symplectic varieties, the general deformation has only been described in a restricted number of cases, each exhibiting some very special and interesting geometry.

In this paper, we stress an unexpected relation between the exceptional groups and certain families of polarized holomorphic symplectic varieties. Briefly, this goes as follows. To each simple complex Lie algebra one can associate a particular projective homogeneous variety $X_{a d}$, which we call the adjoint variety, whose automorphism group is the adjoint form of the Lie group. The adjoint variety is covered by a family of special subvarieties $C$ that we call Legendrian cycles. Then our key observation is that for each exceptional group, there exists a projective variety $X$ of the same dimension as $X_{a d}$, and covered by the same type of special subvarieties, i.e. by Legendrian cycles. Moreover there is a suitable cycle space $P$ for these Legendrian cycles plus certain of their degenerations. And the crucial observation is that for a generic hyperplane section $X_{H}$ of $X$ the subspace $P_{H}$ of $P$ parametrizing the Legendrian cycles $C$ which are contained in $X_{H}$ is a hyperkähler fourfold.

This has to be taken with a grain of salt. Indeed when we start from $F_{4}$, the resulting variety $P_{H}$ is only a blow-up of a hyperkähler fourfold at nine points. Moreover these fourfolds are essentially the same as those that are obtained from $E_{6}$. Starting from $E_{7}$, the related geometry becomes much more complicate, and in the cases $E_{7}$ and $E_{8}$ we had not been able to achieve the expected construction that should follow the above line of the argument. Nevertheless, we believe that its existence also in these two cases remains an interesting challenge.

2000 Mathematics Subject Classification. 14J28, 14J45,14M15.

Key words and phrases. Fano varieties, Hyperkähler varieties, Legendrian varieties, TitsFreudenthal square. 
The following table summarizes the hyperkähler geometries we have encountered.

\begin{tabular}{|c|l|}
\hline$G_{2}$ & Fano variety of lines on a cubic fourfold [1] \\
$D_{4}$ & Hilbert square of determinantal quartic surfaces \\
$F_{4}, E_{6}$ & Zero-locus of a 3-form in 10 variables [5] \\
$E_{7}, E_{8}$ & $? ?$
\end{tabular}

Structutre of the paper.

In Section 2 are collected some facts about the exceptional series of Lie groups, the Tits-Freudenthal magic square and the associated geometries, which are related to our study.

Section 3 provides details on the construction we have just sketched.

Section 4 treats the case of $D_{4}$, for which triality plays an interesting role.

Section 5 discusses the case of $F_{4}$, for which we construct a suitable cycle space for the Legendrian cycle spaces and their degenerations.

In Section 6 we construct the associated hyperkähler fourfold and we show, following a suggestion of Kuznetsov, that it is isomorphic to the fourfold obtained form $E_{6}$.

Acknowledgements. We thank A. Kuznetsov, D. Orlov, K. O'Grady, G. Kaputska and M. Kaputska for their useful hints and comments.

This work has been carried out in the framework of the Labex Archimede (ANR-11LABX-0033) and of the A*MIDEX project (ANR-11-IDEX-0001-02), funded by the "Investissements d'Avenir" French Government programme managed by the French National Research Agency, and the Seoul National University grant 0450-20130016.

\section{The EXCEPtional SERIES And The MAGiC SQUARE}

Let $G$ be a simple complex Lie group, and let $\mathfrak{g}$ denote its Lie algebra. The action of $G$ on $\mathbf{P} \mathfrak{g}$ has a unique closed orbit, which we call the adjoint variety. It is the projectivization of the minimal non trivial nilpotent orbit in $\mathfrak{g}$, and the KostantKirillov-Souriau symplectic structure on this nilpotent orbit descends to a contact structure on the adjoint variety.

Choose a point $x$ of the adjoint variety $X$, and denote by $P$ the stabilizer of $x$ in $G$. Then $P$ is a parabolic subgroup of $G$, and there is an induced isomorphism $X \simeq G / P$. The isotropy representation of $P$ on $T_{x} X$ has a unique invariant hyperplane $V$ (the contact hyperplane), which is (except in type A) an irreducible representation of a given Levi factor of the parabolic group $P$. Recall that a Levi factor is a reductive subgroup of $P$, such that the projection to $P / P^{u}$, the quotient of $P$ by its unipotent radical, is an isomorphism; it is uniquely defined up to conjugation.

Let us denote by $H$ the semisimple part of the chosen Levi factor. Geometrically, the set $C$ of projective lines in $\mathbf{P g}$ contained in $X$ and passing through $x$ is acted on transitively by $H$; its linear span in $T_{x} X$ is the contact hyperplane $V$.

The reduction of $X \subset \mathbf{P g}$ to $C \subset \mathbf{P} V$ is a general process which has been studied in detail in [13]- 15. One of the important aspects of this study is the observation that this reduction process provides a geometric transition between the lines of the Tits-Freudenthal magic square, recently enlarged to a rectangle by Deligne and Gross. Starting from the adjoint varieties of the simple Lie algebras in the Deligne's extended exceptional series, one gets the following list of groups and varieties: 


$\begin{array}{ccccccc}G & G_{2} & S O_{8} & F_{4} & E_{6} & E_{7} & E_{8} \\ h_{G}^{\vee} & 4 & 6 & 9 & 12 & 18 & 30 \\ a & -\frac{2}{3} & 0 & 1 & 2 & 4 & 8 \\ H & S L_{2} & S L_{2}^{3} & S p_{6} & S L_{6} & S_{p i n} & E_{7} \\ C & v_{3} \mathbf{P}^{1} & \left(\mathbf{P}^{1}\right)^{3} & I G(3,6) & G(3,6) & S_{12} & F r\end{array}$

We include in this table the dual Coxeter number $h_{G}^{\vee}$, and the closely related parameter $a=h_{G}^{\vee} / 3-2$. The dual Coxeter number is essentially the parameter used by Deligne in [6]. The parameter $a$ makes more visible the connection with the Tits-Freudenthal magic square, where the Lie algebras of type $F_{4}, E_{6}, E_{7}, E_{8}$ are constructed from the pairs $(\mathbf{A}, \mathbf{O})$, with $\mathbf{A}=\mathbf{R}, \mathbf{C}, \mathbf{H}, \mathbf{O}$ a normed algebra of real dimension $a=1,2,4,8$ - see [13] and the references therein. In particular the existence of the exceptional series appears as a byproduct of the existence of the Cayley (or rather Cayley-Salmon) algebra of octonions. In our study we will meet again the name of Cayley, in an seemingly completely independent, but in fact implicitly connected context: the subgroups $H$ from the series $S p_{6}, S L_{6}, S p i n_{12}, E_{7}$ reappear on the third line of the magic square, where they can be reconstructed from the pairs $(\mathbf{A}, \mathbf{H})$.

A variant of the original Tits-Freudenthal construction, called the triality construction, shows that it is natural to include $\mathrm{SO}_{8}$ in the exceptional series with parameter $a=0$, see [13] and [6, 8]. From this perspective, the group $\operatorname{Spin}_{8}$ and the triality are at the origin of the exceptional series. Finally, $G_{2}$ is deduced from $\mathrm{SO}_{8}$ (or rather by $\operatorname{Spin}_{8}$ ) by a triple folding, and the denominator of the corresponding parameter $a=-2 / 3$ can be thought of as a reminiscence of this folding. The dimensions of $X$ and $V$ as above, and of $C$ from the above table, are connected by the following dimension formulas:

$$
\operatorname{dim} C=3 a+3, \quad \operatorname{dim} V=6 a+8=\operatorname{dim} X-1 .
$$

The fact that the cone $\hat{C}$ over $C$ has half the dimension of $V$ is not a coincidence. There exists an $H$-invariant symplectic structure on $V$ with respect to which $\hat{C}$ is Lagrangian [14]; and $C$, which by itself is a Fano manifold of index $2 a+2$, is said to be Legendrian, see [15], [2] for a systematic study of Legendrian varieties.

\section{LEGENDRIAN CYCLES AND SYMPLECTIC MANIFOLDS}

We suggest a procedure that allows to construct families of holomorphic symplectic manifolds, starting from an adjoint variety $X_{a d}$ of the exceptional series.

First step. Replace $X_{a d}$ by a rational variety $X$ of the same dimension $6 a+9$, covered by cycles of type $C$. This is actually a peculiar step of the construction.

The variety $X$ is again rational and Fano of index $3 a+4$, different from the index of $X_{a d}$, which by itself is $3 a+5$; cf. the tables in $\S 2$ of [4] or in [16] for the invariants for the adjoint varieties of the classical Lie groups. We will denote by $L$ the (very ample) line bundle on $X$ such that $-K_{X}=(3 a+4) L$.

Second step. Find a good parameter space $P$ for cycles of type $C$ in $X$ and their degenerations, which we call Legendrian cycles in $X$. In particular $P$ must be smooth and endowed with a family of cycles $\mathcal{C} \subset P \times X$. 


$\begin{array}{ccccccc}G & G_{2} & S O_{8} & F_{4} & E_{6} & E_{7} & E_{8} \\ X & \mathbf{P}^{5} & \left(\mathbf{P}^{3}\right)^{3} & I G(3,9) & G(3,10) & O G(6,15) & ? ? \\ P & G(2,6) & G(2,4)^{3} & B l G(6,9) & G(6,10) & B l G(12,15) & ? ?\end{array}$

Note that although $P$ is close to be homogeneous, it is not always acted on transitively by the automorphism group of $X$. Two non transitive actions appear for $G=F_{4}$ and $E_{7}$, where $B l$ means that some blowing-up is required. Moreover in the first of these two cases, the automorphism group of $X_{L e g}$ is the odd symplectic group $S p(9)$, which is not semisimple or even reductive. This will be a source of additional technical complications.

The dimension of $P$ is given by the simple formula

$$
\operatorname{dim} P=6 a+12 .
$$

Third step. Let $X_{H}=X \cap H$ be a general hyperplane section of $X$, defined by a general element $h \in H^{0}(X, L)$. In particular $X_{H}$ is smooth of dimension $6 a+8$. We will check that the Hodge cohomology group $H^{3 a+5,3 a+3}\left(X_{H}\right)$ has dimension one, i.e.

$$
H^{3 a+5,3 a+3}\left(X_{H}\right)=\mathrm{C} \Omega
$$

for some closed non-exact $(3 a+5,3 a+3)$-form $\Omega$ on $X_{H}$. Moreover $H^{p, q}\left(X_{H}\right)=0$ for $p+q=6 a+8$ and $q<3 a+3$. In other words, the middle dimensional Hodge structure of $X_{H}$ is of $K 3$ type.

Fourth step. Consider the space $P_{H} \subset P$ of Legendrian cycles $C$ that are contained in $H$. The parameter space $P$ will be constructed in such a way that $E=p_{*} q^{*} L$ will be a rank $6 a+8$ vector bundle on $P$ with $H^{0}(P, E) \cong H^{0}(X, L)$. In particular, $h$ will define a section $h_{E}$ of $E$ with zero-locus $P_{H}=Z\left(h_{E}\right)$, which turns out to be smooth and of dimension four.

Fifth step. Consider the restriction of the family of Legendrian cycles $\mathcal{C} \subset P_{H} \times C_{H}$, with its two projections $p_{H}$ and $q_{H}$. The Abel-Jacobi map

$$
p_{H *} q_{H}^{*}: H^{3 a+5,3 a+3}\left(X_{H}\right) \longrightarrow H^{2,0}\left(P_{H}\right)
$$

maps $\Omega$ to a holomorphic two-form $\omega$ on $P_{H}$ which is generically non-degenerate.

Sixth step. Find, if necessary, a suitable modification $Z_{H}$ of $P_{H}$ with trivial canonical bundle, and conclude that $Z_{H}$ is hyperkähler.

Comments. For $a=-2 / 3,0,2$ the sixth and last step is not necessary: we get directly a hyperkähler structure on $P_{H}$, and no birational modification is needed. Moreover in these cases $P$ appears to be homogeneous. In particular the cycles in $X$ isomorphic to $C$ form a complete family, and there is no need to take degenerations into account.

The hyperkähler vaieties from the case $a=2$ have already been discovered by Debarre and Voisin in [5], where they prove that the result of virtually the same construction is a locally complete family of polarized hyperkähler fourfolds, which are deformations of Hilbert squares of K3 surfaces.

In fact, the case $a=-2 / 3$ is even more classical: it is nothing else than the hyperkähler structure on the Fano variety of lines of a cubic fourfold discovered by Beauville and Donagi in [1].

From our perspective, the special structures of these two very classical varieties have their hidden origin respectively in the exceptional groups $E_{6}$ and $G_{2}$. 
From the triality perspective, the case $a=0$ is the most important. It could have been discovered much earlier, being defined by a very simple and natural vector bundle on a product of three four-dimensional quadrics. It turns out that this construction does not yield a locally complete family of projective hyperkähler manifolds. In fact the symplectic varieties that we obtain are Hilbert squares of determinantal quartic surfaces in $\mathbf{P}^{3}$.

The case $a=1$ will be the object of section 5. As already mentioned, a new feature here is the necessity to take into account degenerations of our Legendrian cycles $C$. The cycle space $P$ will be defined as a blow-up of a Grassmannian. As explained in the fourth step above, it is endowed with a vector bundle, but the zero-locus of its general section is not a hyperkähler manifold. In fact it contains 9 special copies of $\mathbf{P}^{3}$, and it is only after contracting these that we finally obtain a hyperkähler manifold. Alternatively, one can consider, directly on the Grassmannian, the zero-locus of a sheaf which is not locally free.

In the case $a=4$ there is an additional complication: we would expect $X$ to be Fano of index 10, but it is only of index 5 . The reason for this discrepancy is that our Legendrian cycles $C$ in $X$ are copies of $O G_{+}(6,12)$ inside $O G(6,15)$; and the Plücker polarization on $O G(6,15)$, which is certainly primitive, becomes divisible by two when restricted to $O G_{+}(6,12)$, a phenomenon which is directly related to the existence of the Spin representations. So one should modify the construction and ask that the cycle family $\mathcal{C}$ be endowed with a line bundle $\mathcal{L}$ such that $\mathcal{L}^{2}=p^{*} O(1)$, and $E=q_{*} L$ be a vector bundle of rank 32 (given at the generic point of $P$ by a half-spin representation of $\operatorname{Spin}_{12}$ ). This is something we have not managed yet.

The case $a=8$ is the most mysterious, and in this final case we have no suggestion for eventual constructions of $X$ and $P$, which should follow the conjectured Steps $1-6$, as stated above.

An intermediate case, first noticed by Deligne as a complement to the exceptional series is that of $a=6$. Algebraically, one can complete the magic square with the help of the intermediate algebra of sextonions, which is normed for a degenerate quadratic form. Geometrically, we know that the geometries associated with the Freudenthal square can still be constructed from the sextonions, but they yield singular varieties. Therefore it is plausible that our construction may be used for a search of new singular hyperkähler varieties of dimension 4 . If this is indeed the case, the next question will obviously be that of the existence of symplectic resolutions of these fourfolds.

\section{Determinantal quartics AND triality}

In this and the next sections we keep the notation from Section 3, and start with the case $a=0$. The Legendrian cycle corresponding to this case is $C=\mathbf{P}^{1} \times \mathbf{P}^{1} \times \mathbf{P}^{1} \subset \mathbf{P}^{7}$, see e.g. Table I on p. 89 of [2]. Its dual or tangent variety is the quartic hypersurface whose equation is Cayley's famous $2 \times 2 \times 2$ hyperdeterminant [10].

The adjoint variety of $D_{4}$ is the orthogonal Grassmannian $O G(2,8)$, which has dimension $9=6 a+9$ and index $5=3 a+5$, see $\S 2$ in [4]. As already discussed in Section 3, we replace the 9-dimensional adjoint variety of $D_{4}$, which is the orthogonal grassmannian $X_{a d}=O G(2,8)$, by $X=\mathbf{P}^{3} \times \mathbf{P}^{3} \times \mathbf{P}^{3}$. This variety is covered by copies of $C$ parametrized by $P=G(2,4) \times G(2,4) \times G(2,4)$, a triple product of 4 dimensional quadrics. To be more specific we will introduce four-dimensional vector spaces $V_{1}, V_{2}, V_{3}$ and let $X=\mathbf{P} V_{1} \times \mathbf{P} V_{2} \times \mathbf{P} V_{3} . X$ is evidently a Fano manifold of 
dimension $9=6 a+9$ and index $4=3 a+4$ : the anticanonical divisor $-K_{X}=4 L$ for $L=\mathcal{O}_{X}(1,1,1)$.

The parameter space for the Legendrian cycles $C \subset X$ is $P=G\left(2, V_{1}\right) \times G\left(2, V_{2}\right) \times$ $G\left(2, V_{3}\right)$, and $P$ has dimension $6 a+12=12$.

We denote by $T_{i}$ and $Q_{i}$ the rank two tautological bundles and quotient bundles on the Grassmannians $G\left(2, V_{i}\right), i=1,2,3$. The vector bundle $E$ on $P$ of rank $6 a+8=8$ is $E=T_{1}^{*} \otimes T_{2}^{*} \otimes T_{3}^{*}$.

Consider a general element of

$$
H^{0}(X, L)=H^{0}(P, E)=V_{1}^{*} \otimes V_{2}^{*} \otimes V_{3}^{*},
$$

considered either as a section $h$ of $L$, whose zero-locus is a hyperplane section $X_{H}$ of $X$, or as a section $h_{E}$ of $E$, whose zero locus in $P$ is a four-dimensional subvariety $P_{H}$. Note that since $\operatorname{det}(E)=\mathcal{O}(4,4,4)$, the canonical bundle of $P_{H}$ is trivial.

Theorem 1. $P_{H}$ is an irreducible holomorphic symplectic manifold.

One way to show this is to break the symmetry of $h \in V_{1}^{*} \otimes V_{2}^{*} \otimes V_{3}^{*}$, and consider it as a morphism $h_{3}: V_{3} \longrightarrow V_{1}^{*} \otimes V_{2}^{*}$ - or equivalently, a four dimensional space of sections of $\mathcal{O}(1,1)$ on $\mathbf{P}\left(V_{1}\right) \times \mathbf{P}\left(V_{2}\right)$. Generically, such a linear system defines a $\mathrm{K} 3$ surface $S_{3} \subset \mathbf{P}\left(V_{1}\right) \times \mathbf{P}\left(V_{2}\right)$, parametrizing pairs of lines $\left(\ell_{1}, \ell_{2}\right)$ such that $h$ vanishes on $\ell_{1} \otimes \ell_{2} \otimes V_{3}$.

In particular $\ell_{1}$ belongs to the projection of $S_{3}$ to $\mathbf{P}\left(V_{1}\right)$ if and only if the image of $\ell_{1}$ by $h_{1}: V_{1} \longrightarrow V_{2}^{*} \otimes V_{3}^{*} \simeq \operatorname{Hom}\left(V_{2}, V_{3}^{*}\right)$ is generated by a non injective morphism. For $h$ general the rank drops by two only in codimension four, hence nowhere since we are on $\mathbf{P}^{3}$. There the projection of $S_{3}$ to $\mathbf{P}\left(V_{1}\right)$ is in fact an isomorphism to a determinantal quartic surface.

Note also that $\operatorname{Hom}\left(V_{2}, V_{3}^{*}\right) \simeq \operatorname{Hom}\left(V_{3}, V_{2}^{*}\right)$, and that this isomorphism preserves the rank. This implies that if $h$ vanishes on $\ell_{1} \otimes \ell_{2} \otimes V_{3}$, then there exists a line $\ell_{3}$ such that $h$ vanishes on $\ell_{1} \otimes V_{2} \otimes \ell_{3}$. Moreover, this line is unique if $h$ is general. As a consequence, all the three surfaces $S_{1}, S_{2}, S_{3}$ are isomorphic to the same K3 surface $S$, of which we get three in general different representations as a determinantal quartic surface:

$$
Q_{1} \subset \mathbf{P}\left(V_{1}\right), \quad Q_{2} \subset \mathbf{P}\left(V_{2}\right), \quad Q_{3} \subset \mathbf{P}\left(V_{3}\right) .
$$

It is remarkable that this has already been known to Arthur Cayley (see [3] and [9] for more historical information about this classical construction). Cayley has also observed that the correspondence $\ell_{1} \otimes \ell_{2} \otimes V_{3} \mapsto \ell_{1} \otimes V_{2} \otimes \ell_{3}$ can be iterated, and that the iteration

$$
\ell_{1} \otimes \ell_{2} \otimes V_{3} \mapsto \ell_{1} \otimes V_{2} \otimes \ell_{3} \mapsto V_{1} \otimes \ell_{2}^{\prime} \otimes \ell_{3} \mapsto \ell_{1}^{\prime} \otimes \ell_{2}^{\prime} \otimes V_{3}
$$

defines a non-trivial automorphism of $S$. By its construction this automorphism is a byproduct of the triality for $S O(8)$, and so one can call it the triality automorphism.

Theorem 1 will be a consequence of the following more precise statement.

Proposition 1. $P_{H}$ is isomorphic to $\operatorname{Hilb}^{2}(S)$.

Proof. A point of $P_{H}$ is a triple $\left(T_{1}, T_{2}, T_{3}\right)$ in $G\left(2, V_{1}\right) \times G\left(2, V_{2}\right) \times G\left(2, V_{3}\right)$ such that $h$ vanishes on $T_{1} \otimes T_{2} \otimes T_{3}$. This implies that the composition

$$
V_{3} \longrightarrow V_{1}^{*} \otimes V_{2}^{*} \longrightarrow T_{1}^{*} \otimes T_{2}^{*}
$$


has rank at most two, and in fact is equal to two, since for $h$ general it drops to rank one in codimension nine, hence nowhere on the 8-fold $G\left(2, V_{1}\right) \times G\left(2, V_{4}\right)$. The resulting pencil of sections of $\mathcal{O}(1,1)$ on $\mathbf{P}\left(T_{1}\right) \times \mathbf{P}\left(T_{2}\right)$ cuts out a degree two subscheme of $S_{3} \subset \mathbf{P}\left(V_{1}\right) \times \mathbf{P}\left(V_{2}\right)$, which yields a morphism

$$
\delta: P_{H} \longrightarrow H_{i l b}^{2}\left(S_{3}\right)=H_{i l b}^{2}(S) \text {. }
$$

Conversely, a generic point $z$ in $\operatorname{Hilb}^{2}\left(S_{3}\right)$ is represented by two distinct pairs of lines $\left(\ell_{1}, \ell_{2}\right)$ and $\left(\ell_{1}^{\prime}, \ell_{2}^{\prime}\right)$ such that $h$ vanishes on both $\ell_{1} \otimes \ell_{2} \otimes V_{3}$ and $\ell_{1}^{\prime} \otimes \ell_{2}^{\prime} \otimes V_{3}$. Generically the lines $\ell_{1}$ and $\ell_{1}^{\prime}$ are distinct and span a plane $T_{1}$, while $\ell_{2}$ and $\ell_{2}^{\prime}$ span a plane $T_{2}$. The morphism $T_{1} \otimes T_{2} \longrightarrow V_{3}^{*}$ induced by $h$ has rank at most two since it vanishes on $\ell_{1} \otimes \ell_{2}$ and $\ell_{1}^{\prime} \otimes \ell_{2}^{\prime}$, and its image is a two-dimensional space of linear forms on $V_{3}$, defining a plane $T_{3}$. This associates to a generic $z$ in $\operatorname{Hilb}^{2}\left(S_{3}\right)$ a triple $\left(T_{1}, T_{2}, T_{3}\right)$ in $G\left(2, V_{1}\right) \times G\left(2, V_{2}\right) \times G\left(2, V_{3}\right)$, which belongs to $P_{H}$. Then it is straightforward to check that this triple is mapped to $z$ by $\delta$. Therefore $\delta$ must be a birational morphism. But since $P_{H}$ and $H i l b^{2}\left(S_{3}\right)$ both have trivial canonical bundles, the birationality $\delta$ should be an isomorphism.

The fundamental class of $P_{H}$ in $P=G\left(2, V_{1}\right) \times G\left(2, V_{2}\right) \times G\left(2, V_{3}\right)$, as a zero-locus of a section $h_{E}$ of $E=T_{1}^{*} \otimes T_{2}^{*} \otimes T_{3}^{*}$, is $c_{8}\left(T_{1}^{*} \otimes T_{2}^{*} \otimes T_{3}^{*}\right)$. The projection of $P_{H}$ to $G\left(2, V_{1}\right) \times G\left(2, V_{2}\right)$ is an isomorphism with the second degeneracy locus of the induced morphism $V_{3} \longrightarrow T_{1}^{*} \otimes T_{2}^{*}$. By the Thom-Porteous formula, the fundamental class of this degeneracy locus is $c_{2,2}\left(T_{1}^{*} \otimes T_{2}^{*}\right)$, from where by a standard computation one obtains that the degree of $P_{H}$ with respect to the polarization $\mathcal{O}(1,1,0)$ is 432 .

Finally the projection to $G\left(2, V_{1}\right)$ is finite of degree 6 , since a generic fiber can be identified with the set of pairs of points inside the intersection of the quartic surface $Q_{1} \subset \mathbf{P}\left(V_{1}\right)$ with a generic line. Therefore the polarization $\mathcal{O}(1,0,0)$ has degree 12 . It has been shown by Ferretti that $P_{H}$ with this polarization can be deformed to a double EPW sextic, see [7].

\section{The isotropic Grassmannian and its Hilbert scheme}

In this and the next section we discuss the case $a=1$, related to the exceptional group $F_{4}$. The adoint variety $X_{a d}$ for the group $F_{4}$ has dimension $6 a+9=15$ and index $3 a+5=8$, cf. the tables in $\S 2$ of [4]. Following the procedure from Section 3. we will replace $X_{a d}$ by $X=I G_{\omega}(3,9)$, the isotropic Grassmannian defined by a 2 -form $\omega$ of maximal rank on $V_{9}$. This is a Fano variety of the same dimension 15 , and of index 7 . The non semi-simple group $S p(\omega) \simeq S p_{9}$ acts with two orbits on $X$ : 3-planes that contain or do not contain the one-dimensional kernel $K$ of $\omega$.

Following Step 2 in Section 3 , the 15 -fold $X$ is covered by copies of 6 -dimensional Legendre cycles $I G(3,6)$ (see Table I on p. 89 of [2]: the case $F_{4}$, where the Lagrangian Grassmannian $I G(3,6)$ of isotropic 3 -spaces for a non-degenerate 2 -form on $V_{6} \cong \mathbf{C}^{6}$ is denoted by $G_{L}(3,6)$ ), and these Legendre cycles are parametrized by an open subset of $P^{*}=G\left(6, V_{9}\right)$. Indeed to any $V_{6} \subset V_{9}$ one can associate the variety $X \cap G\left(3, V_{6}\right) \subset G\left(3, V_{9}\right)$. This is a copy of $I G(3,6)$ when the restriction of $\omega$ to $V_{6}$ is a non-degenerate 2 -form.

However the restrictions of the general 2-form $\omega$ on $V_{9}$ to some particular subspaces $V_{6} \subset V_{9}$ can be degenerate, which imposes to reconsider the family $P^{*}$ and to replace it with a family $P$ (a birational modification of $P^{*}$ ) which will cover the requirements of Step 2 and the next steps from Section 3 . 
Lemma 1. The action of $S p(\omega)$ on $P^{*}=G\left(6, V_{9}\right)$ has three orbits $O_{i}, i=0,1,2$, defined by the condition that the restriction of $\omega$ to the 6 -space $V_{6} \in P^{*}$ has rank $6-2 i$. The closed orbit $\mathrm{O}_{2}$ has codimension 6 and is isomorphic to $\operatorname{IG}(3,8)$.

Proof. For the general 2-form $\omega$ on $V_{9}$, the corresponding skew-symmetric map $\omega$ : $V_{9} \rightarrow V_{9}^{*}$ has rank 8. If $K \subset V_{9}$ is the 1-dimensional kernel of this map, one can define the 2-form $\omega$ in terms of a non degenerate 2-form $\omega^{\prime}$ on an eight-dimensional space $V_{8}$ identified with the image of the map $\omega$, and a projection $p: V_{9} \rightarrow V_{8}$ from the kernel $K$ of the map $\omega$. Consider some $V_{6} \subset V_{9}$, and let $2 j$ be the rank of the restriction of $\omega$ to $V_{6}$. If $j=0$, then $p\left(V_{6}\right) \subset V_{8}$ is isotropic of dimension at least five, a contradiction. If $j=1$, then $p\left(V_{6}\right)$ contains isotropic hyperplanes, which can have dimension at most four, so $p\left(V_{6}\right)$ has dimension 5 , which means that $V_{6} \supset K$. Moreover by projection, we conclude that $\mathrm{O}_{2}$ can be identified with the subvariety of $G\left(5, V_{8}\right)$ defined by the condition that the rank of the restriction of $\omega^{\prime}$ is minimal. This is the minimal orbit of $\operatorname{Sp}(8)$ inside $G\left(5, V_{8}\right) \simeq G\left(3, V_{8}\right)$, that is the isotropic Grassmannian $I G(3,8)$.

Notation. We denote by

$$
p: P \rightarrow P^{*}
$$

the blowup of $P^{*}=G\left(6, V_{9}\right)$ along the closed orbit $O_{2}$, by $E=p^{-1}\left(O_{2}\right) \subset P$ the exceptional divisor of $p$, and by $\iota: E \rightarrow P$ the embedding of $E$ in $P$.

The variety $P$ is endowed with a natural vector bundle $F$ of rank fourteen, which we describe below.

Since $p: P-E \rightarrow P^{*}-O_{2}=O_{0} \cup O_{1}$ is an isomorphism, over $P-E$ it is enough to define the fibers of $F$ for the 6-spaces $V=V_{6} \in O_{0} \cup O_{1}$.

Denote by $T$ the rank 6 tautological bundle on $P^{*}=G\left(6, V_{9}\right)$ with fiber $T_{V}$ over $V=V_{6}$ identified with the 6-space $V \subset V_{9}$. For $V \in O_{0}$ the restriction $\omega_{V}$ of $\omega$ to $V=T_{V}$ has rank 6 , and by the representation theory of $S p(6)$ the span of the isotropic grassmannian $I G\left(3, T_{V}\right) \cong I G(3,6)$ for $\omega_{V}$ is the projectivization of the 14-dimensional vector space $F_{V}$ which fits in the exact sequence

$$
0 \longrightarrow F_{V} \longrightarrow \wedge^{3} T_{V} \stackrel{\omega_{T}}{\longrightarrow} T_{V} \longrightarrow 0,
$$

where $\omega_{T}$ denotes the contraction with the 2 -form $\omega$. On the orbit $O_{1}$, although the restriction of $\omega$ to $T$ drops rank, the map $\omega_{T}$ remains surjective and the kernel space $F_{V}=\operatorname{Ker}\left(\omega_{T}\right)$ still has dimension 14.

For $V=V_{6} \in O_{2}$, the restriction $\omega_{V}$ of $\omega$ to $V=T_{V}$ has rank two, and the image of $\omega_{T}: \wedge^{2} T_{V} \rightarrow T_{V}=V$ coincides with the 4-dimensional kernel $T_{4} \subset V=T_{V}$ of the skew-symmetric map $\omega_{V}: V \rightarrow V^{*}$. In particular, for $V \in O_{2} \subset P^{*}$ the rank of the kernel $F_{V}$ of $\omega_{T}$ jumps, and so the kernel sheaf of $\omega_{T}$ does not define a vector bundle on $P^{*}=G\left(6, V_{9}\right)$.

Next, we shall see that after performing a blow-up $P \rightarrow P^{*}$ along the closed orbit $\mathrm{O}_{2} \subset P^{*}$, the vector bundle $\mathrm{F}$ on $P^{*}-\mathrm{O}_{2}$ can be completed to a vector bundle on the projective manifold $P$, especially on the exceptional divisor $E$ of the blow-up. Due to the natural isomorphism $P-E \cong P^{*}-O_{2}$ induced by the blow-up, we will keep the notation $F$ also for the vector bundle on $P$.

The normal vector bundle to $O_{2}$ in $P^{*}$ is easily seen to be isomorphic with $\wedge^{2} T_{4}^{\vee}$. Therefore a point of the exceptional divisor of the blow-up, $E \simeq P\left(N_{O_{2} / P^{*}}\right)$, is nothing else than a hyperplane $\Lambda \subset \wedge^{2} T_{4}$. Now it is straightforward to check that for any such 
hyperplane one has $T_{4} \wedge \Lambda=\wedge^{3} T_{4}$. In particular $T \wedge \Lambda$ is of dimension 14 inside $\wedge^{3} T$ (if $T_{2}$ is any complement of $T_{4}$ in $T$, then $T \wedge \Lambda$ is the direct sum of $T_{2} \wedge \Lambda \simeq T_{2} \otimes \Lambda$ of dimension $2 \times 5=10$, and of $T_{4} \wedge \Lambda=\wedge^{3} T_{4}$ of dimension 4 ).

Define the fiber of $F$ over the point $\Lambda$ of $E$ as $F_{\Lambda}:=T \wedge \Lambda$. On the exceptional divisor there is also a bundle $p^{*} T_{4}$, and we denote by $G$ the quotient $p^{*}\left(T / T_{4}\right)$ which has rank two and is self-dual. The above argument yields the following:

Proposition 2. $F$ is a rank fourteen sub-bundle of $p^{*} \wedge^{3} T$ on $P$.

There is an exact sequence

$$
0 \longrightarrow F \longrightarrow p^{*} \wedge^{3} T \stackrel{p^{*} \omega_{T}}{\longrightarrow} p^{*} T \longrightarrow \iota^{*} G \longrightarrow 0 .
$$

Let $h$ denote the pull-back to $P$ of the hyperplane class on $P^{*}$, and $e$ the class of the exceptional divisor $E$.

Corollary 1. $c_{1}(F)=-9 h+2 e$.

Corollary 2. The dual bundle $F^{\vee}$ is globally generated and $H^{0}\left(F^{\vee}\right) \simeq \wedge^{3} V_{9}^{\vee} / \omega \wedge V_{9}^{\vee}$.

Although we will not really need this, it is of worth to mention also the following statement, in accordance with our general approach.

Proposition 3. The projectivization of any fiber of $F$ cuts $X=I G_{\omega}(3,9)$ along a generically reduced, 6-dimensional irreducible subvariety. As a consequence, $P$ parametrizes a family of subvarieties of $X$ made of Legendrian cycles and degenerations of these.

\section{Hyperplane SECTIONS}

Next, we follow Steps 3-6 from Section 3 for the case $a=1$.

A hyperplane section of $X_{H}=X \cap H$ of $X=I G_{\omega}(3,9)$ is defined by a 3 -form $\Omega$ on $V_{9}$, considered up to $\omega \wedge V_{9}$. In this section we study the family of Legendrian cycles $I G(3,6)$, and their degenerations, that are contained in $H$. We denote by $P_{H} \subset P$ the corresponding subvariety. The restrictions of the divisor classes $h$ and $e$ from $P$ to $P_{H}$ will be denoted by $h_{H}$ and $e_{H}$.

Proposition 4. The general 3-form $\Omega$ defines a general section $\tilde{\Omega}$ of $F^{\vee}$, and the zero-locus of $\tilde{\Omega}$ is $P_{H}$. As a consequence, $P_{H}$ is smooth of dimension 4 and its canonical class is $3 e_{H}$.

This suggests to study more carefully the intersection of $P_{H}$ with the exceptional divisor $E$. Our main technical result is the following:

Proposition 5. The intersection $P_{H} \cap E$ is the disjoint union of nine copies of $\mathbf{P}^{3}$.

Proof. Recall that a point $z$ of $E$ is given by a 6-plane $T$ on which $\omega$ has rank two, and a hyperplane $\Lambda \subset \wedge^{2} T_{4}$, where $T_{4} \subset T$ is the kernel of the restriction of $\omega$. Let $\lambda_{4} \subset \wedge^{2} T_{4}^{\vee}$ be a linear form defining $\Lambda$. Choose any lift of $\lambda$ to $\wedge^{2} T^{\vee}$. The orthogonal of $F_{\Lambda}$ in $\wedge^{3} T^{\vee}$ is, independently of the latter choice,

$$
F_{\Lambda}^{\perp}=\wedge^{2} T_{4}^{\perp} \wedge T^{\vee}+\lambda \wedge T_{4}^{\perp} \subset \wedge^{3} T^{\vee} .
$$

Next, we choose a basis $e_{1}, \ldots, e_{9}$ of $V_{9}$ adapted to the situation. We will suppose that $T=\left\langle e_{1}, \ldots, e_{6}\right\rangle$ and $T_{4}=\left\langle e_{1}, \ldots, e_{4}\right\rangle$, so that $T_{4}^{\perp}=\left\langle e_{5}^{*}, e_{6}^{*}\right\rangle \subset T^{*}$ (for simplicity we use the same notation for linear forms on $V_{9}$ and their restrictions to $T$ ). We can 
suppose that the restriction of $\omega$ to $T$ is $e_{5}^{*} \wedge e_{6}^{*}$, and then (after changing the basis if necessary), that

$$
\omega=e_{5}^{*} \wedge e_{6}^{*}+e_{4}^{*} \wedge e_{7}^{*}+e_{3}^{*} \wedge e_{8}^{*}+e_{2}^{*} \wedge e_{9}^{*} .
$$

Clearly, in this basis the 1-dimensional kernel $K$ of $\omega$ is spanned by $e_{1}$. The condition that $z$ belongs to $P_{H}$ means that the restriction of $\Omega$ to $T$ belongs to $F_{\Lambda}^{\perp}$, so that we can write $\Omega$ as

$$
\Omega=e_{5}^{*} \wedge e_{6}^{*} \wedge u^{*}+\lambda \wedge v^{*}+\Omega_{7}^{*} \wedge e_{7}^{*}+\Omega_{8}^{*} \wedge e_{8}^{*}+\Omega_{9}^{*} \wedge e_{9}^{*},
$$

for some 2-forms $\Omega_{7}^{*}, \Omega_{8}^{*}, \Omega_{9}^{*}$, and some linear forms $u^{*}$ and $v^{*}$. Moreover $v^{*}$ is in $T_{4}^{\perp}$, hence it is a linear combination of $e_{5}^{*}$ and $e_{6}^{*}$, and we can suppose that $v^{*}=e_{6}^{*}$. As a conclusion, one is able to write the 2 -form $\omega$ and the 3 -form $\Omega$ as

$$
\begin{gathered}
\omega=e_{5}^{*} \wedge e_{6}^{*}+e_{4}^{*} \wedge e_{7}^{*}+e_{3}^{*} \wedge e_{8}^{*}+e_{2}^{*} \wedge e_{9}^{*}, \\
\Omega=\Omega_{6}^{*} \wedge e_{6}^{*}+\Omega_{7}^{*} \wedge e_{7}^{*}+\Omega_{8}^{*} \wedge e_{8}^{*}+\Omega_{9}^{*} \wedge e_{9}^{*},
\end{gathered}
$$

for some 2-forms $\Omega_{6}^{*}, \Omega_{7}^{*}, \Omega_{8}^{*}, \Omega_{9}^{*}$. More intrinsically, if the 4 -space $W=\left\langle e_{6}^{*}, e_{7}^{*}, e_{8}^{*}, e_{9}^{*}\right\rangle \subset$ $V_{9}^{*}$ is the span of $e_{6}^{*}, \ldots, e_{9}^{*}$, then $\omega \in V_{9}^{*} \wedge W$ and $\Omega \in \wedge^{2} V_{9}^{*} \wedge W$. Note that if the 5 -space $R \in G\left(5, V_{9}\right)$ is the orthogonal to $W$, this means exactly that $\omega$ and $\Omega$ both vanish identically on $R$.

Lemma 2. For a generic pair $(\omega, \Omega)$ of a 2-form $\omega$ and a 3-form $\Omega$ on the 9dimensional space $V_{9}$ there exist exactly nine 5-dimensional subspaces $R_{1}, \ldots, R_{9}$ of $V_{9}$ such that both $\omega$ and $\Omega$ vanish identically on $R_{j}, j=1, \ldots, 9$.

Proof. Denote by $U$ the rank-five tautological vector bundle on the Grassmannian $G\left(5, V_{9}\right)$. The forms $\omega$ and $\Omega$ define general sections of the vector bundles $\wedge^{2} U^{*}$ and $\wedge^{3} U^{*}$ respectively, and the common zero locus of these two sections is the set of 5-spaces $R \subset V_{9}$ on which both $\omega$ and $\Omega$ vanish.

Since the two vector bundles $\wedge^{2} U^{*}$ and $\wedge^{3} U^{*}$ on the 20-dimensional Grassmannian $G\left(5, V_{9}\right)$ are both globally generated and of rank 10, then the zero loci of their generic sections $\omega$ and $\Omega$ intersect in a finite number

$$
N=c_{10}\left(\wedge^{2} U^{*}\right) c_{10}\left(\wedge^{3} U^{*}\right)
$$

of points $R_{j} \in G\left(5, V_{9}\right), j=1, \ldots, N$. It is a standard fact that $c_{10}\left(\wedge^{2} U^{*}\right)$ is the Schubert class $\sigma_{4321}$ defined by the staircase partition. Since this Schubert class is self-dual, $N$ is the coefficient of $\sigma_{4321}$ in $c_{10}\left(\wedge^{3} U^{*}\right)$, expressed in terms of Schubert classes. A computation with Sage finally yields $N=9$.

Let $R_{1}, \ldots, R_{9} \in G\left(5, V_{9}\right)$ be as above, interpreted as 5 -spaces in $V_{9}$. For each $i$, the set of 6-spaces in $V_{9}$ containing $R_{i}$ is a 3 -dimensional projective space $\pi_{i}$ linearly embedded in $P^{*}=G\left(6, V_{9}\right)$. Moreover all these $\pi_{i}^{*}$ are contained in the orbit $O_{2}$ since (as elements of the zero-locus of $\omega$ ) the 5-spaces $R_{i} \subset V_{9}$ are isotropic with respect to $\omega$, see Section 5. If $p: P \rightarrow P^{*}=G\left(6, V_{9}\right)$ is the blowup of $O_{2}$ as in Section 5, and $P_{H} \subset P$ is the family of Legendrian cycles in $X_{H}$ as above (cf. also with Step 4 in Section 3), then by the preceding:

$$
p\left(P_{H} \cap E\right) \subset \pi_{1}^{*} \cup \cdots \cup \pi_{9}^{*} .
$$

We claim that each $\pi_{i}$ can be lifted to a 3 -space $\pi_{i}$ in $E$, and that

$$
P_{H} \cap E=\pi_{1} \cup \cdots \cup \pi_{9} .
$$


Indeed, once we have written the 3 -form $\Omega$ as

$$
\Omega=\Omega_{6}^{*} \wedge e_{6}^{*}+\Omega_{7}^{*} \wedge e_{7}^{*}+\Omega_{8}^{*} \wedge e_{8}^{*}+\Omega_{9}^{*} \wedge e_{9}^{*}
$$

in the adapted basis $e_{1}, \ldots, e_{9}$ as in the beginning of this section, with corresponding 5 -space, say $R_{1}=\left\langle e_{1}, \ldots, e_{5}\right\rangle$, and its orthogonal $W_{1}=\left\langle e_{6}^{*}, e_{7}^{*}, e_{8}^{*}, e_{9}^{*}\right\rangle$, we get a welldefined map

$$
\bar{\Omega}: V_{9} / R_{1} \longrightarrow \wedge^{2} R_{1}^{\vee}
$$

which is injective for the general pair $(\omega, \Omega)$. The map $\bar{\Omega}$ associates to any 6 -space $T$ containing $R_{1}$ a line in $\wedge^{2} R_{1}^{\vee}$, which by restriction is mapped to a line in $\wedge^{2} T_{4}^{\perp}$. This defines the lifting of $\pi_{1}^{*}$ to $\pi_{1} \subset p^{-1}\left(\pi_{1}\right) \subset E$.

Theorem 2. The nine divisors $\pi_{1}, \ldots, \pi_{9}$ in $P_{H}$ can be contracted. The resulting variety $Z_{H}$ is a smooth irreducible hyperkähler fourfold.

Proof. First observe that the lift $\pi_{i}$ of $\pi_{i}^{*}$ to $E$ being linear, the restriction of $\mathcal{O}_{E}(1)$ to $\pi_{i}$ coincides with the pull-back of $\mathcal{O}_{P^{*}}(1)$, and also with $\mathcal{O}_{\pi_{i}}(1)$. This implies that the canonical class of $\tilde{\pi}_{i}$ is $-4 e_{\pi_{i}}$, and since the canonical class of $P_{H}$ is $3 e_{H}$ we get that the normal bundle of $\pi_{i}$ in $P_{H}$ is $\mathcal{O}_{\pi_{i}}(-1)$. Therefore the divisor $\pi_{i} \subset P_{H}$ can be contracted to a smooth point for any $i=1, \ldots, 9$. Let

$$
f: P_{H} \rightarrow Z_{H}
$$

be the composition of the 9 contractions, where by $Z_{H}$ we have denoted the image variety $Z_{H}=f\left(P_{H}\right)$. By the preceding, $Z_{H}$ is a smooth projective variety of dimension $4=\operatorname{dim}\left(P_{H}\right)$. By the formulas for blowups, the canonical classes of $P_{H}$ and $Z_{H}$ are connected by the formula

$$
3 e_{H}=K_{P_{H}}=f^{*} K_{Z_{H}}+3\left(\pi_{1}+\cdots+\pi_{9}\right)=f^{*} K_{Z_{H}}+3 e_{H} .
$$

Therefore $f^{*} K_{Z_{H}}$ is trivial, which implies that the canonical class $K_{Z_{H}}$ by itself is trivial, and as we will see below $Z_{H}$ is in fact a holomorphic symplectic fourfold.

In order to see the last, consider a 10-dimensional space $V_{10}$ containing our $V_{9}$ as a hyperplane, and choose a vector $v_{0} \in V_{10}-V_{9}$. There is an induced dual decomposition $V_{10}^{*}=V_{9}^{*} \oplus \mathbf{C} v_{0}^{*}$, where $v_{0}^{*}$ is the linear form evaluating to zero on $V_{9}$ and to one on $v_{0}$. Correspondingly, at the level of three-forms we get the decomposition $\wedge^{3} V_{10}^{*}=\wedge^{3} V_{9}^{*} \oplus \wedge^{2} V_{9}^{*} \wedge v_{0}^{*}$. In particular, our 2-form $\omega$ and 3-form $\Omega$ on $V_{9}$ define a 3-form $\Omega_{0}=\Omega+\omega \wedge v_{0}^{*}$ on $V_{10}$. By [5], the subvariety $P_{H_{0}} \subset G\left(6, V_{10}\right)$ parametrizing 6 -planes on which $\Omega_{0}$ vanishes identically, is a holomorphic symplectic fourfold. The following statement will conclude the proof of the Theorem.

Proposition 6. $Z_{H}$ is isomorphic to $P_{H_{0}}$.

Proof. The projection of $\mathbf{P}\left(V_{10}\right)$ to $\mathbf{P}\left(V_{9}\right)$ from $\left\langle v_{0}\right\rangle$ induces a rational map

$$
\pi: G\left(6, V_{10}\right) \longrightarrow G\left(6, V_{9}\right) .
$$

This rational map is defined outside the locus $G_{0}$ of 6 -dimensional subspaces of $V_{10}$ containing $v_{0}$, which is a copy of $G\left(5, V_{9}\right)$ and has codimension 4 in $G\left(6, V_{10}\right)$.

Given a 6-dimensional subspace $T \subset V_{9}$, a subspace $T_{0} \subset V_{10}$ such that $\pi\left(T_{0}\right)=T$ can be described as the space of vectors $x+u(x) v_{0}, x \in T$ for some linear form $u$ on $T$. Moreover, the fact that $\Omega_{0}$ vanishes identically on $T_{0}$ is equivalent to the vanishing of $\Omega+u \wedge \omega$ on $T$. This implies that $\pi$ maps $P_{H_{0}}$ birationally to $P_{H}$. We denote the restriction of $\pi$ by $\pi_{H}: P_{H_{0}} \longrightarrow P_{H}$. 
The rational map $\pi$ can be resolved by just blowing-up $G_{0} \simeq G\left(5, V_{9}\right)$. Note that $\Omega_{0}$ vanishes on $T_{0}=\mathbf{C} v_{0} \oplus U$ if and only if $\Omega$ and $\omega$ both vanish on $R$. So the (schematic) intersection of $G_{0}$ with $P_{H}$ can be identified with the set of nine points $R_{1}, \ldots, R_{9}$ in $G\left(5, V_{9}\right)$ defined in Lemma 2 . We can resolve $\pi_{H}$ by blowing up these nine points, which yields a birational morphism $\tilde{\pi}_{H}: \tilde{P}_{H_{0}} \longrightarrow P_{H}$. Moreover the nine components of the exceptional divisor are then mapped linearly, hence isomorphically, to $\pi_{1}, \ldots, \pi_{9}$. In particular the birational morphism $\tilde{\pi}_{H}$ preserves the canonical class, so it must be an isomorphism. It only remains to contract the nine 3-spaces on both sides to get an induced isomorphism $\bar{\pi}_{H}: P_{H_{0}} \longrightarrow Z_{H}$.

\section{REFERENCES}

[1] Beauville A., Donagi R., La variété des droites d'une hypersurface cubique de dimension 4, C. R. Acad. Sci. Paris, Sér. I Math. 301 (1985), 703-706.

[2] Buczynski J., Legendrian Subvarieties of Projective Space, Geom. Dedicata, Vol. 118 issue 1 (2006), 87-103.

[3] Cayley A., A memoir on quartic surfaces, Proc. London Math. Soc. 3 (1869-71), 19-69.

[4] Chaput P.E., Perrin N., On the quantum cohomology of adjoint varieties, Proc. London Math. Soc. (3) 103 (2011), 294-330.

[5] Debarre O, Voisin C., Hyper-Kähler fourfolds and Grassmann geometry, J. Reine Angew. Math. 649 (2010), 63-87.

[6] Deligne P., La série exceptionnelle de groupes de Lie, C.R.A.S.322 (1996), 321-326.

[7] Ferretti A., Special subvarieties of EPW sextics, Math. Z. 272 (2012), no. 3-4, 1137-1164.

[8] Deligne P., Gross B., On the exceptional series, and its descendants, C.R.A.S. 335 (2002), $877-881$.

[9] Festi D., Garbagnati A., van Geemen B., van Luijk R., The Cayley-Oguiso automorphism of positive entropy on a K3 surface, J. Mod. Dyn. 7 (2013), 75-97.

[10] I. M. Gelfand, M. M. Kapranov, A. V. Zelevinsky, Discriminants, Resultants, and Multidimensional Determinants, Birkhäuser, 2008.

[11] Iliev A., Manivel L., Fano manifolds of CalabiYau Hodge type, J. Pure Appl. Algebra 219 (2015), no. 6, 2225-2244.

[12] Iskovskih V., Prokhorov Y., Fano varieties, in Algebraic geometry V, 1247, Encyclopaedia Math. Sci. 47, Springer 1999.

[13] Landsberg J.M., Manivel L., Triality, exceptional Lie algebras and Deligne dimension formulas, Adv. Math. 171 (2002), 59-85.

[14] Landsberg J.M., Manivel L., The projective geometry of Freudenthal's magic square, J. Algebra 239 (2001), 477-512.

[15] Landsberg J.M., Manivel L., Legendrian varieties, Asian J. Math. 11 (2007), 341-359.

[16] Snow D., Homogeneous vector bundles, available at www3.nd.edu/ snow/Papers/HomogVB.pdf.

Department of Mathematics, Seoul National University, Gwanak Campus, Bldg. 27, SEOUL 151-747, KOREA

Email address: ailiev@snu.ac.kr

Institut de Mathématiques de Marseille, UMR 7373 CNRS/Aix-Marseille Université, Technopôle Château-Gombert, 39 Rue Frédéric Joliot-Curie, 13453 MARSEILle Cedex 13, France

Email address: laurent.manivel@math.cnrs.fr 